
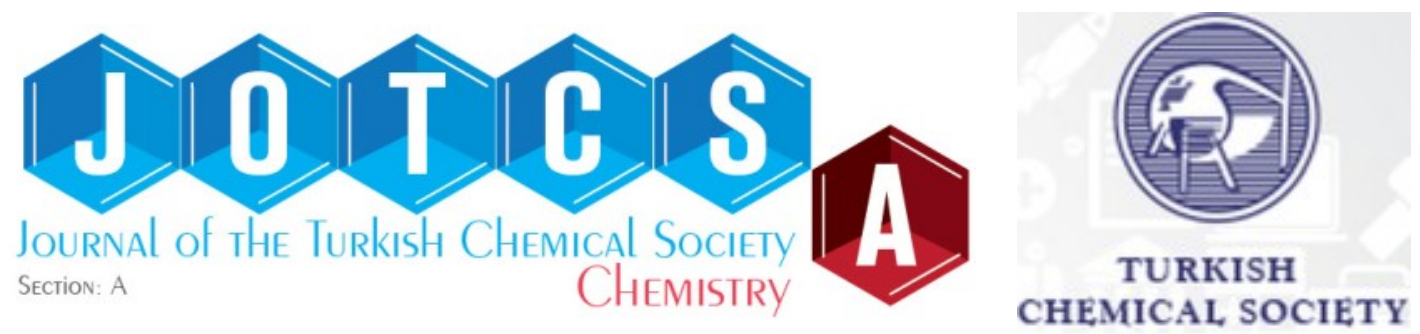

\title{
Understanding Inhibition of Steel Corrosion by Some Potent Triazole Derivatives of Pyrimidine through Density Functional Theory and Molecular Dynamics Simulation Studies
}

\author{
Bello Abdullahi Umar* $\triangle(D)$, Adamu Uzairu $\square$ and Gideon Adamu Shallangwa $\square$ \\ Department of Chemistry, Ahmadu Bello University, Zaria, Nigeria
}

\begin{abstract}
Density Functional Theory (DFT) calculation at B3LYP level of theory and 6-31G* basis set was applied on some triazole derivatives of pyrimidine which led to the optimization of their structures, generation of electronic and other important Quantum chemical descriptors such as the energy of the highest occupied molecular orbital ( $E_{\text {номо }}$ ), the energy of the lowest unoccupied molecular orbital ( $E_{\text {Lumo }}$ ), energy band gap $(\Delta E)$, Dipole Moment $(\mu)$, chemical hardness $(\eta)$, chemical softness $(\sigma)$, global electronegativity $(X)$ and number of transferred electrons $(\Delta N)$ using SPARTAN'14 Software. The obtained results shows a good correlation between the chemical structures of the inhibitors and their experimental inhibition efficiencies (\%IEs). The ranking of these efficiencies (\%IEs) nicely matched with the order of a good number of the generated descriptors but with a varying degree of correlation as majority of the descriptors indicates that I4 is the best inhibitor among the data set. Furthermore, molecular dynamic (MD) simulations were carried out to search the best adsorption configuration of the inhibitor on the steel (llll $\left.1 \begin{array}{ll}1 & 0\end{array}\right)$ surface using Material Studio 8.0. The obtained results of MD simulations suggest that the interaction was as a results of the chemical adsorption on the steel surface, since the binding energy $>100 \mathrm{Kcalmol}^{-1}$ for all the inhibitors and the best adsorption energy was found to be $-488.07 \mathrm{Kcalmol}^{-1}$ (I-4). This observation are in good agreement with the DFT results and the experiment findings. Thus; this study provides a valuable approach and new direction to novel steel corrosion inhibitor discovery.
\end{abstract}

Keywords: Triazole Derivatives of pyrimidine; DFT (B3LYP/6-31G*); MD simulation.

Submitted: July 23, 2018. Accepted: October 03, 2019.

Cite this: Umar B, Uzairu A, Shallangwa G. Understanding Inhibition of Steel Corrosion by Some Potent Triazole Derivatives of Pyrimidine through Density Functional Theory and Molecular Dynamics Simulation Studies. JOTCSA. 2019;6(3):455-66.

DOI: https://doi.org/10.18596/jotcsa.446084.

*Corresponding author. E-mail: abdallahbum@yahoo.com.

\section{INTRODUCTION}

Corrosion of steel is a serious mechanical problem which affects several areas of industrial processes especially the oil industry which always leads to enormous economic losses $(1,2)$. The corrosion of steel may cause mechanical damage of equipment and lead to changes in the chemical properties of processing substances. The cost of corrosion globally reported in 2010 was about \$2.2 Trillion, which was around $3 \%$ of $\$ 73.33$ Trillion (world's entire domestic product) $(3,4)$. Prevention of corrosion exhibited a vital role for many industries particularly petrochemical industries that often make use of steel (2). Although it is not possible to 
completely avoid the corrosion process, there are various method to prevent it or slow down its rate $(5,6)$. One of the most economical and simple methods was the use of organic compounds especially those that contain $\mathrm{O}, \mathrm{N}, \mathrm{P}$, and $\mathrm{S}$ heteroatoms and also pi-electron systems as corrosion inhibitors $(7,8)$.

Experimental techniques like weight-loss method, EIS (electrochemical impedance spectroscopy), potentiodynamic polarization, etc. $(9,10)$, have been used to understand the corrosion process and its inhibition. Although experiments mostly are time-consuming, costly, and lacking in explaining the mechanism of inhibition of the corrosion (11, 12). Thus, quantum chemical calculation method was endorsed as a potent and easy tool to reduce the cost and time and can help in the interpretation of the experimental findings $(13,14)$. Additionally, Molecular dynamics (MD) simulation was recently considered as a new tool to study the adsorptive behavior of the corrosion inhibitors on a metallic surfaces of interest $(11,15)$.

Furthermore, the corrosion inhibition efficiency of a series of a new set of triazole derivatives of pyrimidine was synthesized lately by Gonzalez-
Olvera et al (16). The required products were found to have high yields and characterized by mass spectroscopy, IR and NMR. These synthesized compounds were evaluated as inhibitors for steel corrosion in $1 \mathrm{M} \mathrm{HCl}$ solution employing EIS method. The findings shows the effective behavior of these compounds as inhibitors for steel corrosion in acidic solution. To the best search of the authors, these observations was not studied with any theoretical method. Thus, in the present study, quantum chemical calculations and molecular dynamics simulations were carried out on these compounds in order to give a clear relationship between their electronic structures, geometrical parameters and the binding energies with their experimental inhibition efficiencies (\%Es) earlier reported by Gonzalez-Olvera et al (16).

\section{COMPUTATIONAL DETAILS}

\section{Selection of Inhibitors}

A set of ten triazole derivatives as corrosion inhibitors were selected from the literature (16) and used for this present study. The 2D structure of each inhibitor in the data set was drawn using ChemDraw ultra V12.0. The structures of the inhibitors and their experimental inhibition efficiency (\%IE) are presented in Table 1.

Table 1. Triazole Derivatives of Pyrimidine Nucleobases and Their Inhibition Efficiency (\%IE)

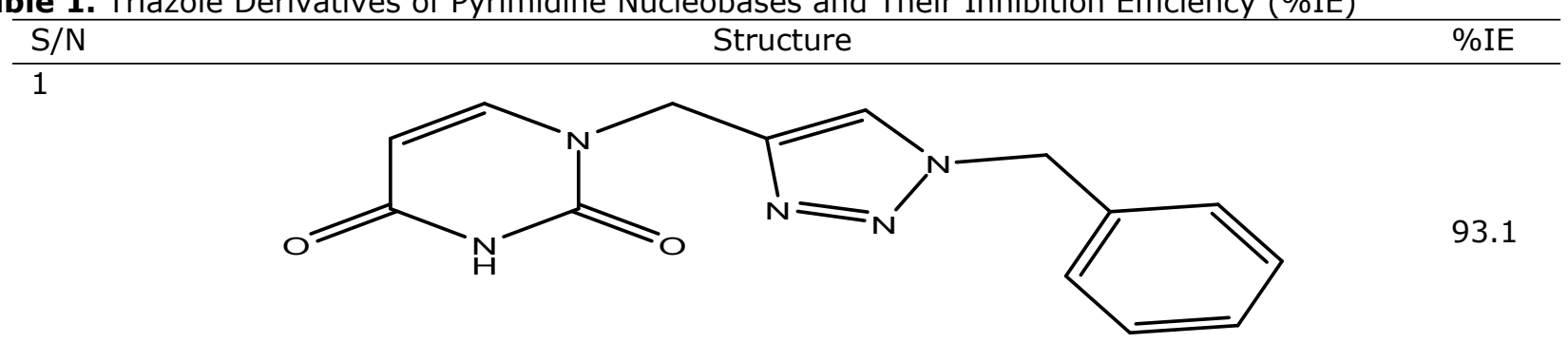

2

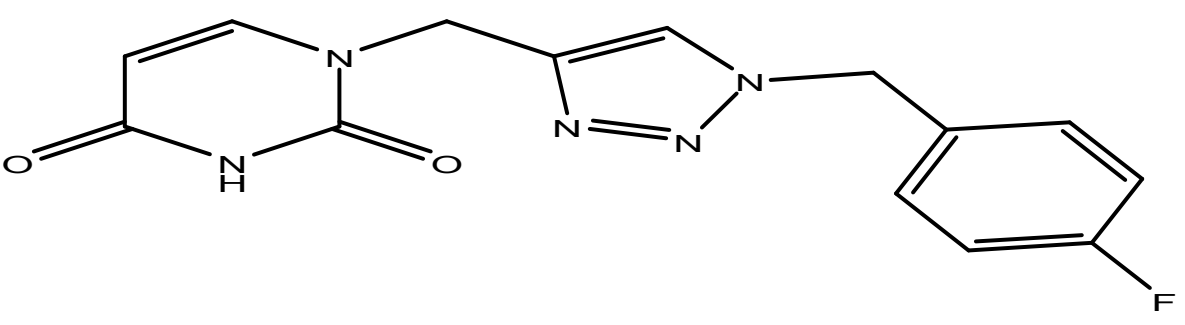

95.6 
Umar BA, Uzairu A, Shallangwa GA. JOTCSA. 2019; 6(3): 451-462.

3

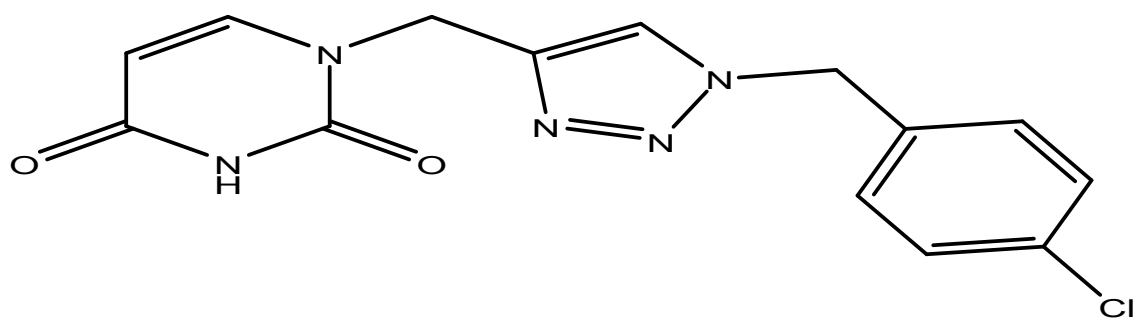

4

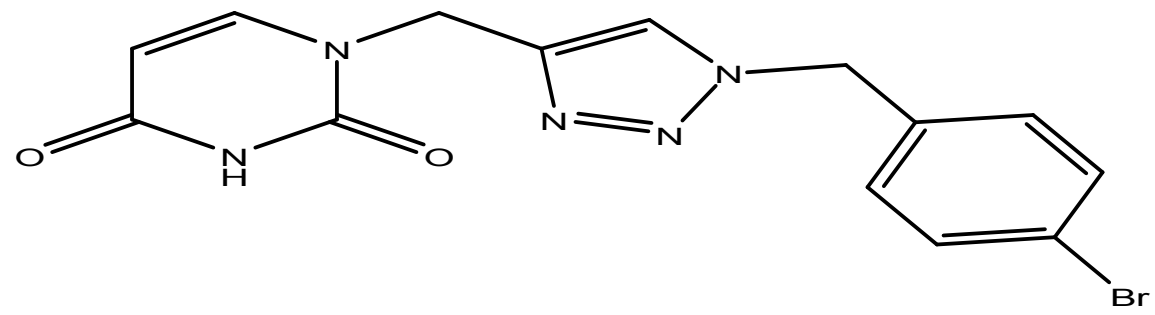

96.1

5

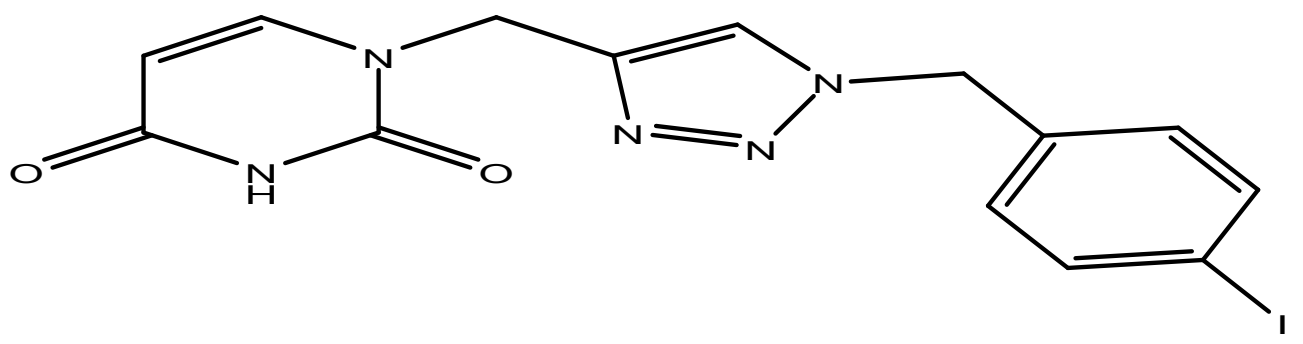

Table 1. (continued)

$\mathrm{S} / \mathrm{N}$<smiles>CCn1cc(C)c(=O)[nH]c1=O</smiles>

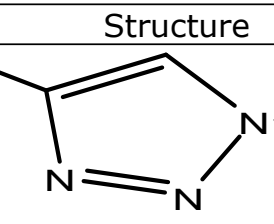<smiles>C1CCCCC1</smiles>

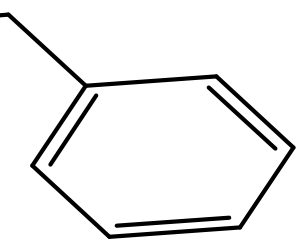

7<smiles>Cc1cn(Cc2cn[nH]n2)c(=O)[nH]c1=O</smiles><smiles>Fc1ccc(CI)cc1</smiles>

90.2

95.0 

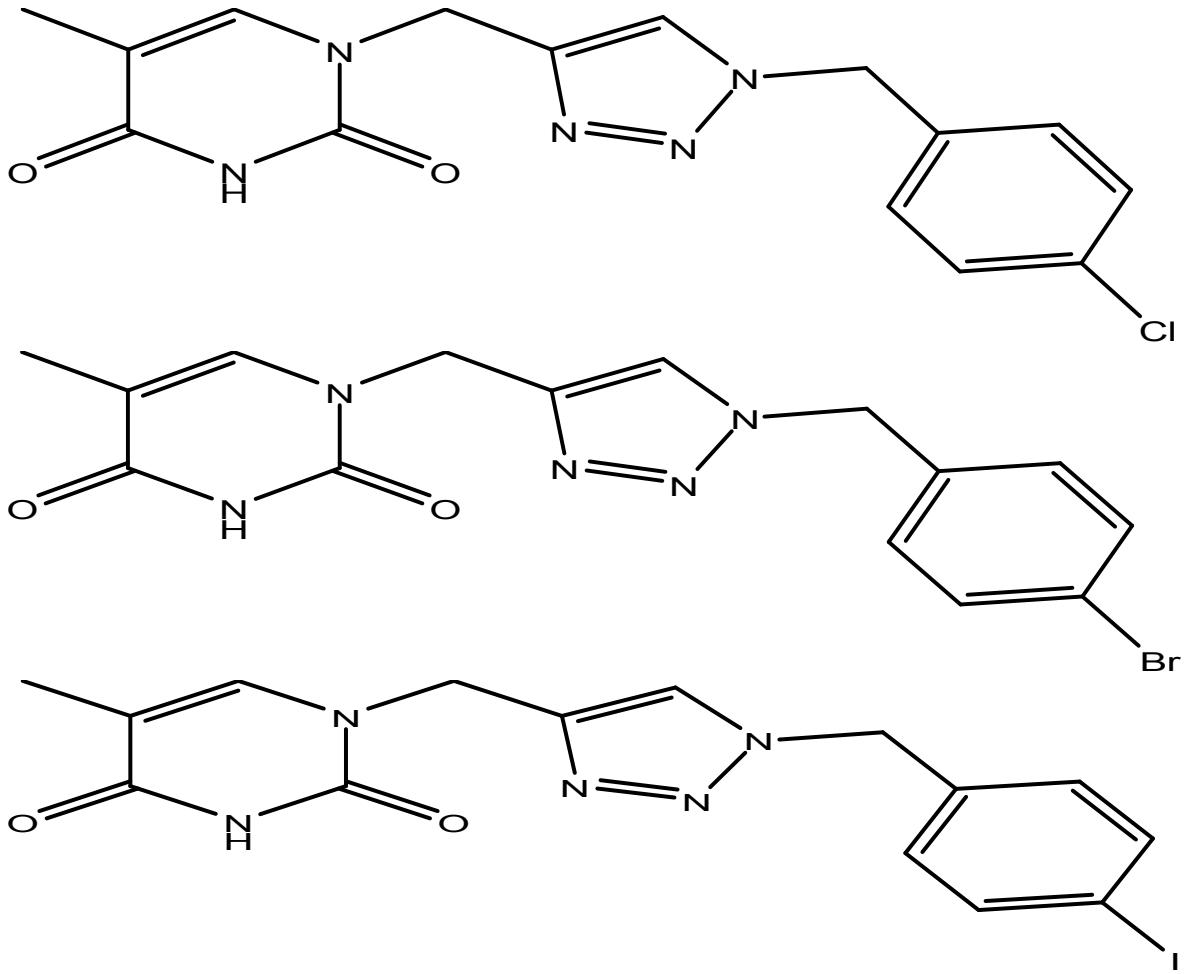

\section{Optimization and calculation of chemical parameters}

The 2D structure of each inhibitor was converted to the 3D structure using Spartan 14.0 software. The molecular mechanic force field $(\mathrm{MM}+)$ was used to clean and minimize the structures using of SPARTAN'14 software on Dell Intel(R)Core(TM)i7$5500 U$ CPU), $16.00 \mathrm{~GB}$ RAM @ $2.400 \mathrm{GHz} 2.400 \mathrm{GHz}$ processor on Windows 8.1 Pro 64-bit Operating system, $\times 64$-based processor, so as to remove all the strain on the structures of the inhibitor molecules. Additionally, this guarantee a fullydefined and stable conformation of the studied inhibitor molecules of this study $(17,18)$. Geometry optimization was set at ground state utilizing the DFT at the B3LYP level of theory and 6-311G (d) basis set. The generated chemical parameters were energy of highest occupied molecular orbital (E-HOMO), energy of lowest unoccupied molecular orbital (E-LUMO), dipole moment $(\mu)$ and some others were calculated such as energy band gap $(\Delta E)$, chemical hardness $(\eta)$, chemical softness $(\sigma)$, global electronegativity $(X)$, number of transferred electrons $(\Delta N)$ using relevant relations (Equations 1-5) as reported before in literature $(5,15)$.

$$
\begin{aligned}
& \Delta E=E_{L U M O}-E_{\text {HOMO }} \\
& \eta=\frac{-1}{2}\left(E_{\text {HOMO }}-E_{L U M O}\right)
\end{aligned}
$$

$$
\begin{gathered}
\sigma=\frac{1}{\eta}=-\left(\frac{2}{\left(E_{H O M O}-E_{L U M O}\right)}\right) \quad \text { (Eq. 3) } \\
\chi=\frac{-\left(E_{H O M O}+E_{L U M O}\right)}{2} \quad \text { (Eq. 4) } \\
\sigma=\frac{1}{\eta}=-\left(\frac{2}{\left(E_{H O M O}-E_{L U M O}\right)}\right) \\
\Delta N=\frac{\left(\chi_{F e}-\chi_{i n h}\right)}{2\left(\eta_{F e}+\eta_{i n h}\right)} \quad(\mathrm{Eq} .5) \\
\text { Where } \mathrm{XFe}_{\mathrm{Fe}}=7 \mathrm{eV} \text { and } \eta_{\mathrm{Fe}}=0(19)
\end{gathered}
$$

\section{Molecular Dynamics (MD) simulation}

The simulation (MD) was applied to portray the adsorption mechanism between the studied inhibitors and the steel surface using Materials studio version 8.0 material simulation program from Biovia. The inhibitors were optimized and modeled employing COMPASS force field. The system (simulation) was carried out at $298 \mathrm{~K}$ using Andersen thermostat with NVE microcanonical ensemble at a time step of $1.0 \mathrm{fs}$ and the simulation time was set as $5 \mathrm{ps}$. The simulation was conducted in a box (simulation-box) of (24.82 $\AA$ $\times 24.82 \AA \times 45.27 \AA$ ) under periodic boundaries conditions. The simulation box contains a Fe (110) slab, solvent (acid) molecules $\left(\mathrm{H}_{2} \mathrm{O}+\mathrm{H}_{3} \mathrm{O}^{+}+\mathrm{Cl}^{-}\right)$and 
an inhibitor. Fe (110) surface was chosen as the studied Fe surface, because Fe (110) was the most density packed Fe surface and stable(20,21). The Fe crystal has ten (10) layers out of which seven (7) down layers were frozen. The adsorption and binding energies values were calculated using equation 6 and 7 respectively (15).

$$
\begin{array}{cc}
E_{\text {adsorption }}=E_{\text {Total }}-\left(E_{\text {Surface }}+\mathrm{H}_{2} \mathrm{O}+\mathrm{H}_{3} \mathrm{O}^{+}+\mathrm{Cl}^{-}+E_{\text {Inhibitior }}\right) & \text { (Eq. 6) } \\
E_{\text {binding }}=-E_{\text {adsorption }} & \text { (Eq. 7) }
\end{array}
$$

Where $\mathrm{E}_{\text {Total }}$ is, $E_{\text {Surface }}+\mathrm{H}_{2} \mathrm{O}+\mathrm{H}_{3} \mathrm{O}^{+}+\mathrm{Cl}^{-}+$Inhibitor (the total energy of the corrosion system) and $-E_{\text {surface }}+$ $\mathrm{H}_{2} \mathrm{O}+\mathrm{H}_{3} \mathrm{O}^{+}+\mathrm{Cl}^{-}$is the energy of the Fe surface together with $\mathrm{H}_{2} \mathrm{O}+\mathrm{H}_{3} \mathrm{O}^{+}+\mathrm{Cl}^{-}$molecules and Einhibitor is the energy of the inhibitor.

\section{RESULTS AND DISCUSSIONS}

Quantum parameters of the studied inhibitors In order to examine the inhibitive performance of the inhibitors in relation to the molecular structures, some DFT (Quantum) parameters were generated to explain their mode of adsorption. The geometry optimized molecular structure, the distributions of HOMO and LUMO of the first inhibitor are depicted in Figure 1. Table 4 present the results of DFT parameters of the studied inhibitors like the E-HOMO, E-LUMO, Dipole Moment $(\mu)$, Energy band gap $(\Delta \mathrm{E})$, chemical hardness $(\eta)$, chemical softness $(\sigma)$, global electronegativity $(X)$ and number of transferred electrons $(\Delta N)$ computed using Spartan 14 software at B3LYP level of theory, 6-31G $(d, p)$ basis set and some relevant equations.

The HOMOs of the studied inhibitors are mainly dominated on the pi-bond and then delocalized over the whole aromatic pyrimidine ring in each molecule as seen in supplementary Table 1 . The halogen substituents in each of the inhibitors from I-2 to I-10 are also involved in the HOMO. Pyrimidine ring of inhibitor 1 in Figure 1 shows a significant contribution of the two nitrogen atoms, pi-bond and the two oxygen atoms of the -dione to the distributions of HOMO and LUMO. The HOMO electron density distribution of the studied inhibitors indicate favorable interactions of the inhibitors with an electron lacking metallic orbitals. The distribution of the LUMO electron densities of all the studied inhibitors were also spread all over on the pyrimidine rings. The electronic surfaces of HOMO and LUMO revealed the pyrimidine ring has the potential to donate and accept electrons under favorable conditions. This typical behavior is suitable for donor-acceptor interactions which may be responsible for the adsorption of inhibitors on the steel surface.

Based on the frontier orbital (molecular) approximation, the donor/acceptor relation occur in between HOMOs and LUMOs (frontiers (molecular) orbitals) of reacting molecules $(22,23)$. The adsorption mechanism of an inhibitor on a metallic surface usually increases with the increase of the HOMO energy and decreases with the decrease of LUMO energy. Therefore, the inhibitor molecule can donate their lone pair of electrons from the HOMO orbital to the $d$-orbital of the metallic molecule and also the inhibitor molecule will receive the electrons coming from the metallic $d$-orbital through the LUMO orbital for in-electron-donation and electronback-donation method. Therefore, E-HOMO has always related with electron donation of an inhibitor; a high value of E-HOMO shows the high tendency of an inhibitor molecule to donate lone pair of electrons to the d-orbital of the acceptor metal. 
A
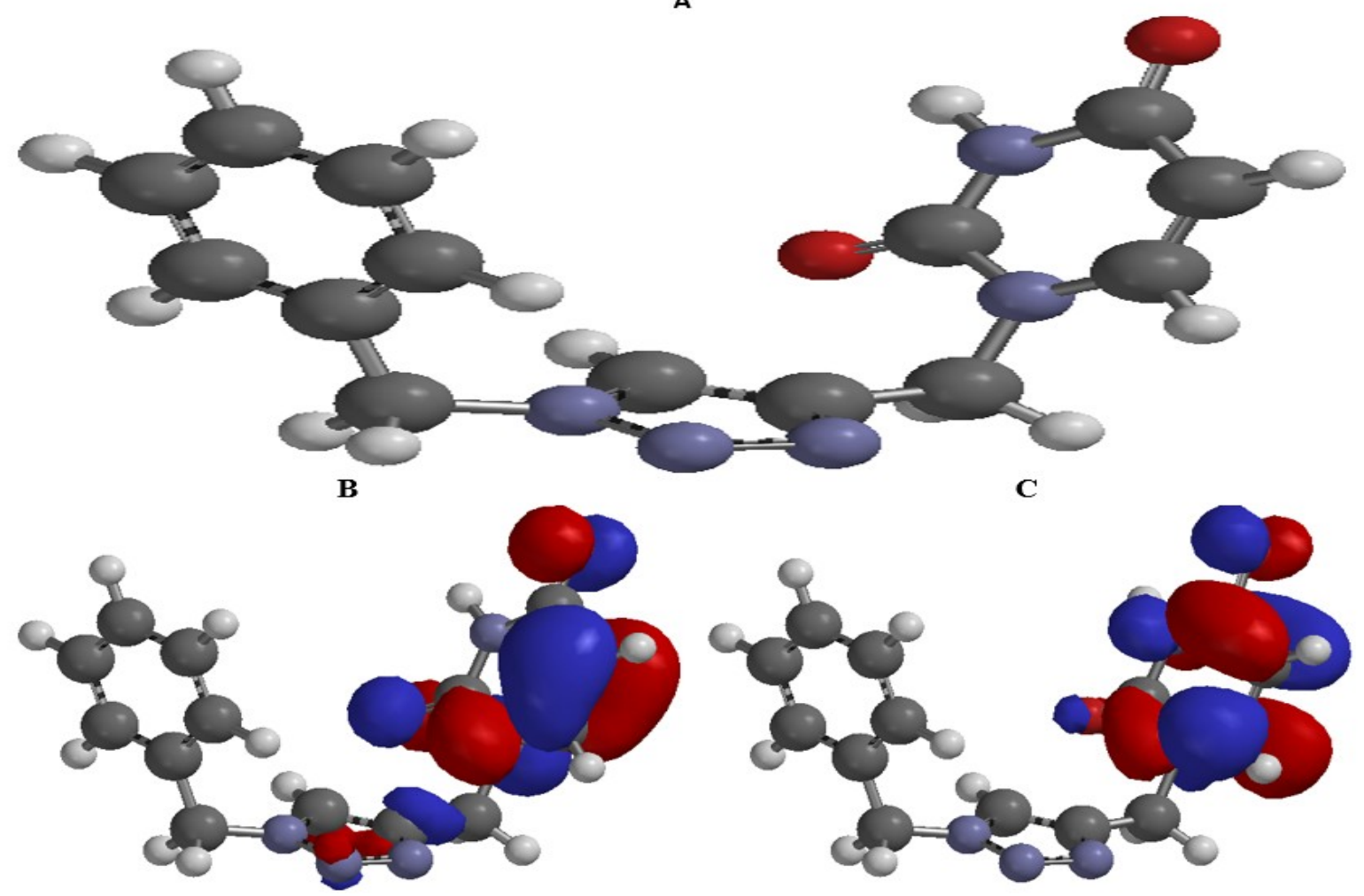

Figure 1. (A) Optimized Structure (B) HOMO distribution and (C) LUMO distribution for I-1(Inh.1).

Table 2. Selected quantum chemical parameters of the studied inhibitors.

\begin{tabular}{|l|l|l|l|l|l|l|l|l|}
\hline $\mathrm{S} / \mathrm{N}$ & $\mathrm{E}-\mathrm{HOMO}(\mathrm{eV})$ & $\mathrm{E}-\mathrm{LUMO}(\mathrm{eV})$ & $\Delta \mathrm{E}$ & $\mu$ (Debye) & $\eta$ & $\sigma$ & $\mathrm{X}$ & $\Delta \mathrm{N}$ \\
\hline 1 & -6.59 & -1.04 & 5.55 & 3.38 & 2.775 & -0.360 & 3.815 & 4.419 \\
\hline 2 & -6.63 & -1.07 & 5.56 & 3.85 & 2.780 & -0.359 & 3.850 & 4.379 \\
\hline 3 & -6.66 & -1.10 & 5.56 & 4.04 & 2.780 & -0.359 & 3.880 & 4.337 \\
\hline 4 & -6.66 & -1.09 & 5.57 & 4.03 & 2.785 & -0.359 & 3.875 & 4.352 \\
\hline 5 & -6.65 & -1.08 & 5.57 & 3.79 & 2.785 & -0.359 & 3.865 & 4.365 \\
\hline 6 & -6.32 & -0.90 & 5.42 & 3.99 & 2.710 & -0.369 & 3.610 & 4.593 \\
\hline 7 & -6.38 & -0.95 & 5.43 & 3.13 & 2.715 & -0.368 & 3.665 & 4.527 \\
\hline 8 & -6.39 & -0.96 & 5.43 & 2.01 & 2.715 & -0.368 & 3.675 & 4.513 \\
\hline 9 & -6.41 & -0.98 & 5.43 & 1.92 & 2.715 & -0.368 & 3.695 & 4.487 \\
\hline 10 & -6.40 & -1.09 & 5.31 & 2.06 & 2.655 & -0.377 & 3.745 & 4.321 \\
\hline
\end{tabular}

Energy of highest occupied molecular orbital (E-HOMO), Energy of lowest unoccupied molecular orbital (E-LUMO) Energy band gap ( $\triangle E$ ), dipole moment $(\mu)$ chemical hardness $(n)$, chemical softness $(\sigma)$,global electronegativity $(X)$, number of transferred electrons $(\Delta N)$.

From Table 2, the values of E-HOMO for the studied inhibitors increases in the order I-4 $>\mathrm{I}-3>$ $\mathrm{I}-5>\mathrm{I}-2>\mathrm{I}-1>\mathrm{I}-9>\mathrm{I}-10>\mathrm{I}-8>\mathrm{I}-7>\mathrm{I}-6$ respectively. The trend in the increase of the HOMO energies show some level of consistency with the increase of the efficiencies (inhibition), Thus, I-4 with a high efficiency $(96.1 \%)$ has a larger HOMO energy and I-6 with the lowest efficiency $(90.2 \%)$ has a lower HOMO energy as seen in Table 2. Conversely, a low value of E-LUMO of an inhibitor molecule demonstrate the high tendency of the inhibitor molecule to receive electrons coming from the metallic d-orbital during the back-donation process. From Table 2, the ELUMO value in the ascending order are: I- $6>\mathrm{I}-7>$ I-8 $>$ I-9 $>$ I-1 $>$ I-2 $>$ I-5 $>$ I-4 $>$ I-10 $>$ I-3. This indicates that I-3 has a stronger chances of accepting electrons from orbital of the metal as they interact with the Fe surface. Therefore, I-3 may strongly adsorb on the surface of the metallic and achieve better inhibition effectiveness though the second highest in terms of \%IE. I-6 strongly 
agrees with EIS findings as it has the lowest \%IE having the highest LUMO energy.

Energy band gap, $\Delta \mathrm{E}$ is another crucial property as most efficient inhibitors were characterized by a small value of $\Delta \mathrm{E}(24)$. In Table 2, the trend at which the energy gap decreases is not consistent with the increase in the experimental \%IEs of the studied inhibitors. However, the reverse is the case i.e. the $\Delta \mathrm{E}$ increases when the \%IEs decreases. The dipole moment $(\mu)$ is another parameter which is commonly used for the prediction of direction of the inhibition process. It shows a distribution of the electrons on the molecule, and also measure the polarity in any given bond (25). The molecules with higher $\mu$ values tend to form a strong interactions (dipole-dipole) with the metallic steel surface, resulting to a strong adsorption of the inhibitor on the metallic surface and hence resulting in the better IE\% of an inhibitor (26). Nevertheless, there is no established correlation between the $\mu$ and IE $\%$ reported (21). The results shown in Table 2 indicate that I-3 has the largest $\mu$ value and I-9 has the lowest $\mu$ value. Among all the ten (10) studied inhibitors, increasing the $\mu$ leads to an increment of their IE\% due to the increase in the intermolecular forces.

Inhibitor with the large hardness ( $\eta$ ) value is expected to be weaker compared to other inhibitors with lower values (7). Thus, as shown in Table 2, the hardness value increases as the \%IE increases. I-4 has the highest $\eta$ value hence the best inhibitor and this agrees with the experimental observation reported in table 1 . The larger $\sigma$ value indicates that the inhibitor have softer nature and will have higher chances of donating electrons to the metallic molecule (27). The arrangement across the inhibitors in the $\sigma$ values as presented in Table 2 decreases in the order, I-2 $>$ I-3 $>$ I- $4>$ I$5>\mathrm{I}-1>\mathrm{I}-7>\mathrm{I}-7>\mathrm{I}-8>\mathrm{I}-9>\mathrm{I}-6>\mathrm{I}-10$. This suggests that I- 2 has the highest and therefore the most reactive inhibitor molecule.

The electronegativity $(x)$ describes the electron attracting power of an inhibitor molecule. The high value of $x$ shows the strong attracting capability of an inhibitor molecule to accept the electrons from the metallic (Fe) surface (11). Afterward, the inhibitors that have larger electronegativities would have better interaction with Fe surface and then will have better IE\%. From Table 2, it can be observed that the $x$ values of the ten inhibitors obey the order I- $3>\mathrm{I}-4>\mathrm{I}-5>\mathrm{I}-2>\mathrm{I}-1>\mathrm{I}-10$ $>\mathrm{I}-9>\mathrm{I}-8>\mathrm{I}-7>\mathrm{I}-6$, hence, it is confirmed that I-3 followed by I-4 has the higher tendency of accepting electrons and I- 6 has the least among the studied inhibitors and these findings are in good agreement with the experimental findings. The values of $\Delta N$ demonstrate the fraction of electrons that transfer from the inhibitor to the metallic surface. If $\Delta \mathrm{N}>0$, then electrons was transferred from the inhibitor molecule to the Fesurface while if $\Delta N<0$, then electrons were transferred from Fe-surface to inhibitor molecule through electron back donation process (15). From Table 2, it can be observed that the values of $\Delta N$ of the studied inhibitors are all greater than $0(\Delta N>$ $0)$, this indicates that all the studied inhibitors may donate electrons on to the Fe surface through the formation of a coordinate covalent bond. Examination of the results in Table 2 indicates that the values of $\Delta N$ of the inhibitors has a very little difference. This further confirms the electron donating ability of the studied inhibitors as observed from their experimental findings.

\section{Molecular Dynamic Simulation Studies}

Electronic parameters alone are not enough to determine the trend of the inhibitive performances of these studied inhibitors despite its success in explaining the mechanism of action of the inhibitors. Thus, it is essential to conduct thorough modelling of the direct interactions of the studied inhibitors with the Fe surface. It is believed that the major mechanism of the inhibition of corrosion is through adsorption. Therefore, adsorption of these inhibitors on the steel (Fe) surface was simulated to predict the nature of interactions between the studied inhibitors and Fe (110) crystal surface in $1 \mathrm{M} \mathrm{HCl}$. The first step of this simulation process was the geometry optimization of the studied inhibitors, solvent (acid) molecules $\left(\mathrm{H}_{2} \mathrm{O}\right.$, $\mathrm{H}_{3} \mathrm{O}^{+}$, and $\mathrm{Cl}^{-}$) and $\mathrm{Fe}(110)$ crystal. The geometry optimization of the corrosion system was conducted until the total energy of the system reached a local minimum on the potential energy surface. During the optimization process, the atomic coordinates were adjusted using COMPASS force-field which continues unless the total energies of the individual structures reached to the minimum energy, after which, a box was created for the simulation process by importing the $\mathrm{Fe}$ (110) crystal, solvent molecules $\left(\mathrm{H}_{2} \mathrm{O}, \mathrm{H}_{3} \mathrm{O}^{+}\right.$and $\mathrm{Cl}^{-}$) and the inhibitor molecule. Then the MD process were conducted and the system attained equilibrium when both the energy and temperature of the system were balanced. All the other inhibitors were studied similarly. The equilibrium configurations of the simulated system for the first inhibitor are depicted in Figure 4. It could be observed that in Figure 5 and 6 at the middle of the MD simulation process the system moves towards the equilibrium. After the system attained equilibrium, the adsorption and the binding energy values of the inhibitors adsorbed on the Fe surface 
was calculated according to equations (6) and (7)

respectively and are presented in Table 3.

Table 3. Molecular Dynamic Simulations Results of the studied inhibitors.

\begin{tabular}{|l|l|l|l|l|l|l|}
\hline $\mathrm{S} / \mathrm{N}$ & $\mathrm{E}_{\mathrm{Fe}+\text { soln }}\left(\mathrm{kcalmol}^{-1}\right)$ & $\mathrm{E}_{\text {Inh. }}\left(\mathrm{kcalmol}^{-1}\right)$ & $\mathrm{E}_{\text {Total }}\left(\mathrm{kcalmol}^{-1}\right)$ & $\mathrm{E}_{\text {Ads. }}\left(\mathrm{kcalmol}^{-1}\right)$ & $\mathrm{E}_{\text {Bind. }}\left(\mathrm{kcalmol}^{-1}\right)$ & $\mathrm{BD}(\AA)$ \\
\hline 1 & -125832.513 & -42.694 & -126294.487 & -419.28 & 419.28 & 2.595 \\
\hline 2 & -125832.513 & -40.231 & -126346.164 & -473.42 & 473.42 & 2.891 \\
\hline 3 & -125832.513 & -40.376 & -126353.079 & -480.19 & 480.19 & 2.390 \\
\hline 4 & -125832.513 & -40.323 & -126360.906 & -488.07 & 488.07 & 2.160 \\
\hline 5 & -125832.513 & -40.284 & -126316.487 & -443.69 & 443.69 & 3.18 \\
\hline 8 & -125832.513 & -78.975 & -126350.438 & -438.95 & 438.95 & 2.920 \\
\hline 9 & -125832.513 & -78.997 & -126377.5 & -465.99 & 465.99 & 2.783 \\
\hline 9 & -125832.513 & -75.505 & -126389.708 & -481.69 & 481.69 & 2.842 \\
\hline 10 & -125832.513 & -72.986 & -126383.419 & -477.92 & 477.92 & 2.780 \\
\hline
\end{tabular}

$E_{\text {Fe+soln. }}$ (Energy of $\mathrm{Fe}+\mathrm{H}_{2} \mathrm{O}+\mathrm{H}_{3} \mathrm{O}^{+}+\mathrm{Cl}^{-}$), $E_{\text {Inh. }}$ (Energy of the inhibitor), $E_{\text {Total }}($ total energy of the simulation system), $E_{\text {Ads. }}$ (Adsorption Energy), $E_{\text {Bind. }}$ (Binding Energy), BD (Bond Distance).

It can be observed from the molecular structures of the examined inhibitors that these molecules contain various electrons (lone pair) on the $\mathrm{N}$ and $\mathrm{O}$ atoms and also n-bonds of the aromatic systems. Therefore, these electrons (lone pair) on the heteroatoms will be donated to an empty d orbitals of Fe metal. It could be observed in Figure 4, that the inhibitor adsorbs almost flatly in orientation with the Fe surface and the interaction is more through the pyrimidine ring. This flat orientation of the inhibitor molecule on the $\mathrm{Fe}$ surface can be as a result of a chemical bond formation between the inhibitor and Fe surface. This observation was supported by the HOMO and LUMO distributions of these inhibitors as the region (pyrimidine ring) was found to be the most active center of adsorption (see Figure 1). Additionally, it could be seen from the results presented in Table 3 that the values of the adsorption energy of the simulation systems at $298 \mathrm{~K}$ were large and negative. These high negative values of adsorption energies may be attributed to the strong interaction which occur between these inhibitors and Fe surfaces. Thus, the calculated adsorption energy values reveal that inhibitor 4 adsorbs on the Fe surface more spontaneously than any other inhibitor among the series. However, the adsorption capability of an inhibitor molecule on the Fe surface may also be determined from the values of the binding energy. The larger the binding energy is, the better will be the adsorption. Therefore, it is further confirmed from the results presented in Table 3, that all the adsorption of the inhibitors on the Fe surfaces occurred through chemical adsorption process since the magnitude of the binding energies is actually within the range of chemisorption (>100 kcal mol-1) (28). 


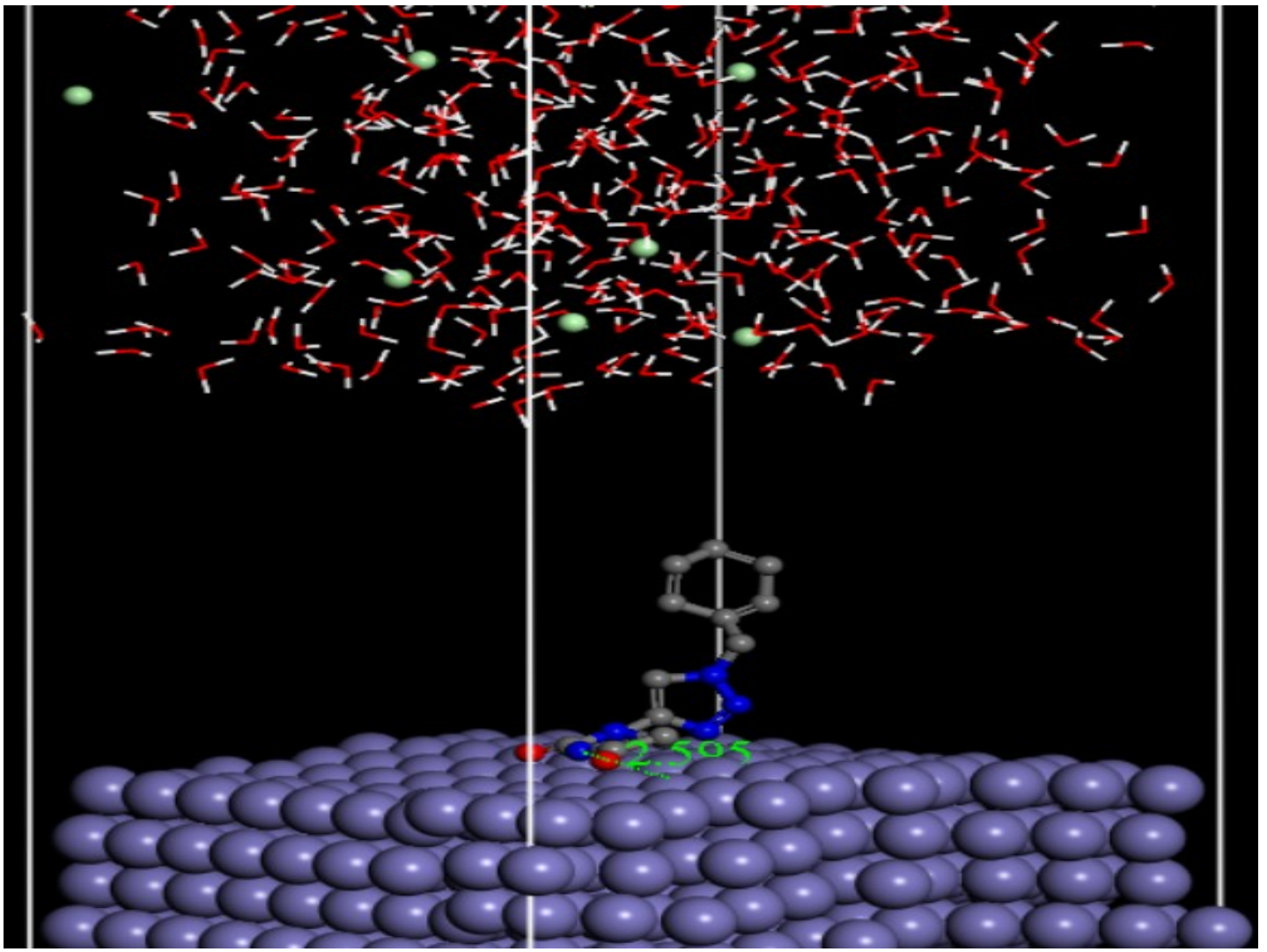

Figure 2. Equilibrium interaction configurations of the first inhibitor on Fe $\left(\begin{array}{lll}1 & 1 & 0\end{array}\right)$ surface.

Forcite Dynamics Energies

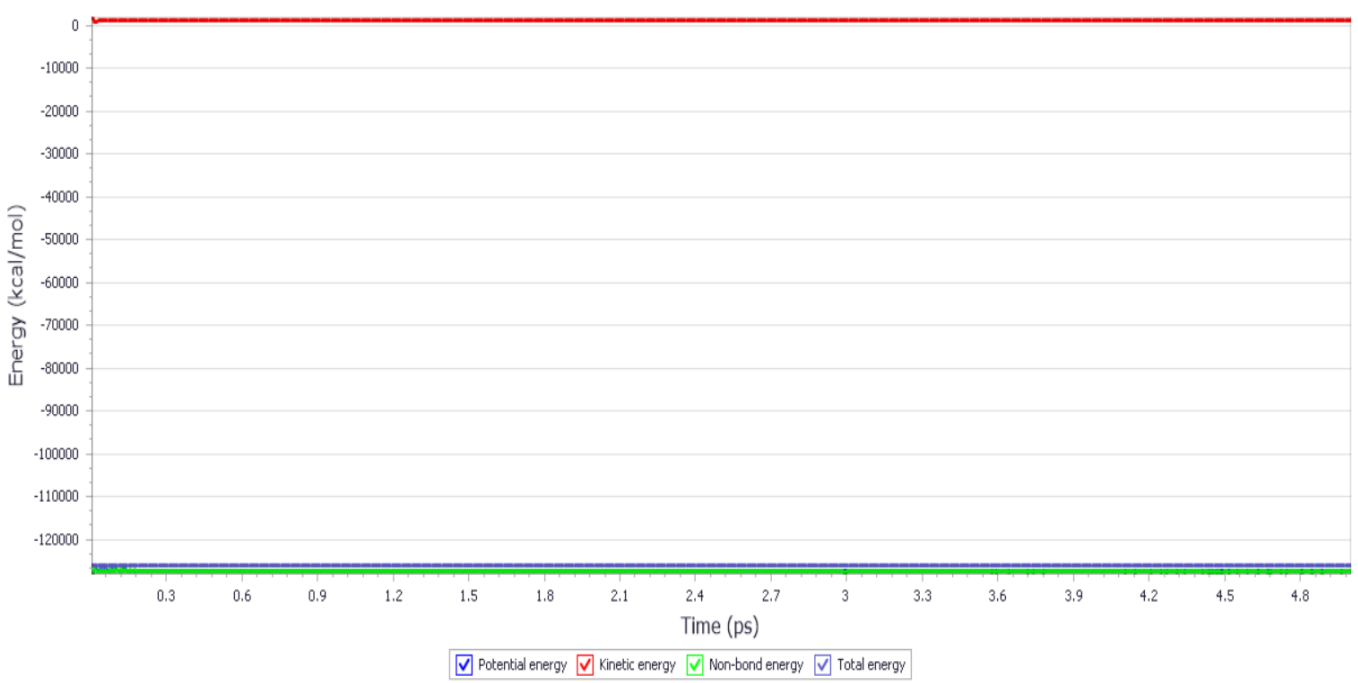

Figure 3. Fluctuation curves of Energies obtained from MD simulation for the first inhibitor. 


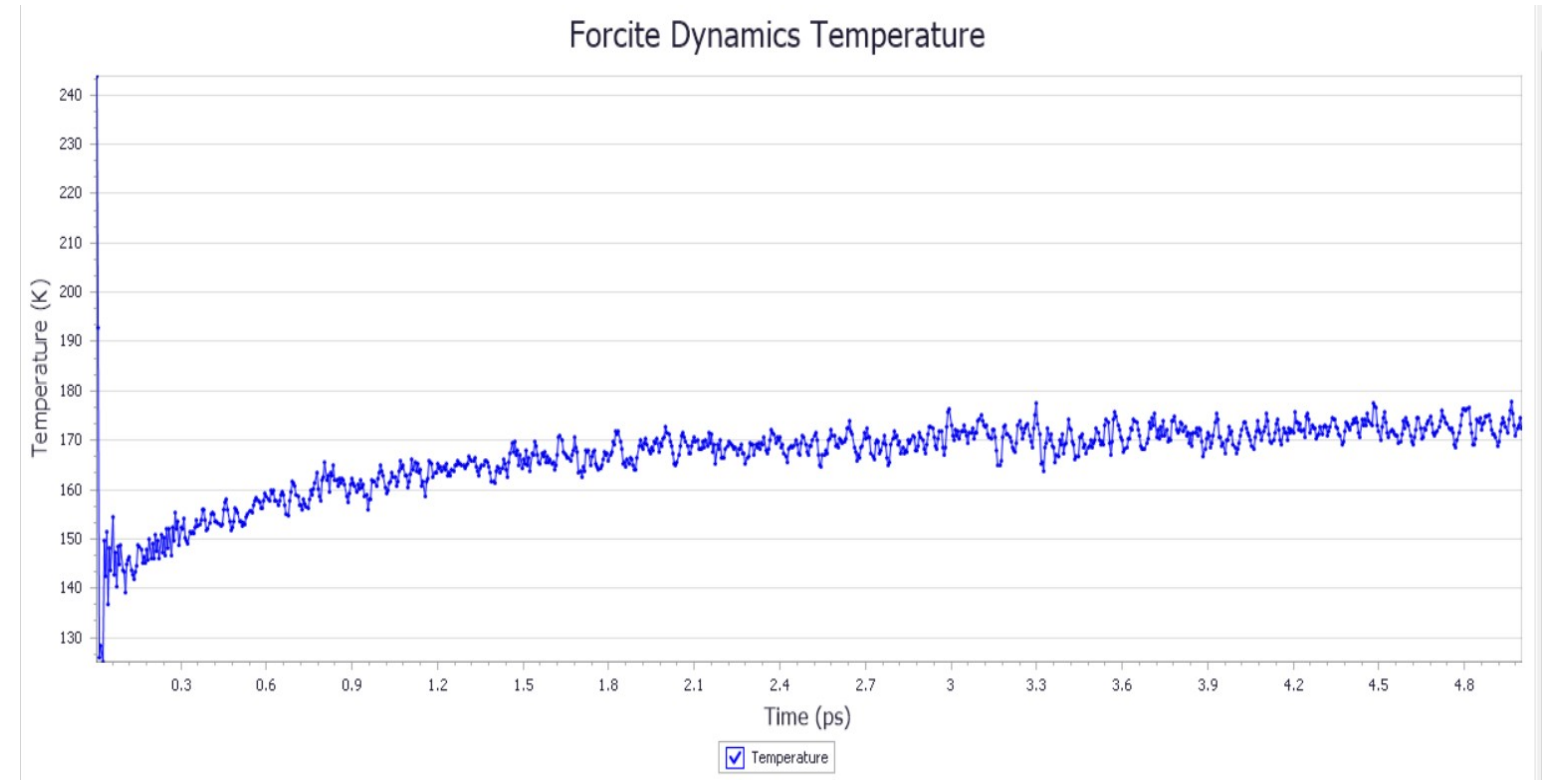

Figure 4. Equilibrium Temperature curve recorded from MD simulation for the first inhibitor.

Generally, bond distance measured within $3.5 \AA$ describes a strong chemical bond formation between the atoms and the bond distance greater than $3.5 \AA$ signifies interaction between the atoms are of Van der Waals type $(29,30)$. Figure 4 indicates the bond distance (shortest) between the $\mathrm{N}$-atom of the pyrimidine ring of the first inhibitor and Fe surface. The measured bond distances for all the studied inhibitors and Fe-surface were shown in table 3 . From the mentioned values in Table 3, it can be clearly seen that all the measured bond distances are in the range $(3.5 \AA)$, this indicates that chemical bond was formed between these inhibitors and the Fe surface. Thus, chemical adsorption occurred on Fe surfaces. These findings are consistent with the experimental results obtained from EIS measurement (16) as well as from quantum chemical studies. This could be explained from the molecular structures of the studied inhibitors and the distribution of the HOMOs and LUMOs. Inhibitors having halogen atoms in their molecular structures are more and easily adsorbed on metallic surfaces and shows better efficiency (inhibition). It was clear from the structures given in Table 1 that inhibitor $1-5$ has the similar pattern with the introduction of halogen atoms which increases the inhibition efficiency in the order $\mathrm{Br}>\mathrm{Cl}>\mathrm{F}$ while the iodine atom slightly decreased the \%IE. For that reason, inhibitor-4 with bromine atom as the substituent was the best corrosion inhibitor among the studied inhibitors. Similarly, inhibitor 6-10 had a similar pattern with $\mathrm{F}, \mathrm{Cl}$ and $\mathrm{Br}$ and methyl group as substituent have the same effect in increasing the inhibition efficiency while the increase with iodine as the substituent is also slightly lower. In this regard the inhibitors with $\mathrm{Br}, \mathrm{F}$ or $\mathrm{Cl}$ are regarded to be the most potential corrosion inhibitors for steel.

Furthermore, in order to check the affection of the solvent (acidic) solution on the adsorption process of the system, the adsorption behavior of the first inhibitor in vacuum was simulated via MD simulation. The adsorption energy obtained in acidic solution is much less than those obtained in the vacuum. This could be due to the less influential effect in vacuum and the complex nature of the corrosion process in solution, like adsorption of the solvent (acid) molecules, the affection of the acid solution, most especially the anions, that have been proved to have a high influence on adsorption of the inhibitor (8). It was found that the adsorption energy of this inhibitor $(-1811.528 \mathrm{kcal}$ $\mathrm{mol}^{-1}$ ) changed significantly compared with the one simulated in acidic solution $\left(-419.28 \mathrm{kcal} \mathrm{mol}^{-1}\right)$. So also, the adsorption behavior is not consistent to those in the acid solution. Therefore, considering a solvent in the molecular dynamic simulation process is very necessary.

\section{CONCLUSION}

It has been clearly shown that the approach employed in this study was successful in explaining the inhibition performance of the studied inhibitors. Quantum chemical calculation shows that the reactive sites in the structure of the inhibitors are majorly the $\mathrm{N}$-atom of the pyrimidine ring, $\mathrm{n-}$ electron centers, and a halogen substituent. Electron-donation and electron-acceptance 
capability of these inhibitors were consistent with the results obtained from the EIS experiment. The interaction energies ( $E_{\text {ads. }}$ and $E_{\text {bind. }}$ ) between the inhibitors and Fe $\left(\begin{array}{lll}1 & 1 & 0\end{array}\right)$ metal surface via MDsimulation were large indicating chemical bond formation and that the inhibitors can more tightly adsorb on the iron surface. Inhibitor 4 showed high adsorption energy $\left(-488.07 \mathrm{kcal}^{\mathrm{mol}^{-1}}\right)$ among other co-inhibitors as observed from the experiment. From this molecular dynamics simulations analysis, we realized that the order of values of the adsorption and binding energy were in good agreement to the experimental findings and also the shortest bond distances measured indicates that these inhibitors have a very strong interactions with steel (Fe) surface. Thus, this quantum chemical calculations and molecular dynamic simulation results correlate with each other and suggest the directions for the design of new anti-corrosion molecules with better inhibitive potential toward corrosion of steel.

\section{ACKNOWLEDGMENTS}

The authors sincerely acknowledge Ahmadu Bello University, Zaria for providing the softwares used and all the members of the group for their advice and encouragement in the cause of this research.

\section{REFERENCES}

1. Szklarska-Smialowska Z, ZS-Smialowska. Pitting and crevice corrosion: NACE International Houston, TX; 2005.

2. Singh P, Ebenso EE, Olasunkanmi LO, Obot I, Quraishi M. Electrochemical, theoretical, and surface morphological studies of corrosion inhibition effect of green naphthyridine derivatives on mild steel in hydrochloric acid. The Journal of Physical Chemistry C. 2016;120(6):3408-19.

3. Obot IB. Recent advances in computational design of organic materials for corrosion protection of steel in aqueous media. Developments in corrosion protection: InTech; 2014.

4. Al Hashem A. Corrosion in the Gulf Cooperation Council (GCC) states: statistics and figures. proceedings of the Corrosion $U A E, A b u$ Dhabi, UAE. 2011.

5. Nwankwo HU, Ateba CN, Olasunkanmi LO, Adekunle AS, Isabirye DA, Onwudiwe DC, et al. Synthesis, characterization, antimicrobial studies and corrosion inhibition potential of 1,8-dimethyl$1,3,6,8,10$, 13-hexaazacyclotetradecane: experimental and quantum chemical studies. Materials. 2016;9(2):107.
6. Usmana B, MOHAMMED AS, Umarb A. QUANTUM CHEMICAL EVALUATION ON CORROSION INHIBTION PERFORMANCE OF BALANITIN-7 ON MILD STEEL IN 1M HYDROCHLORIC ACID SOLUTION. Applied Journal of Environmental Engineering Science.4(3):4-3 (2018) 380-386.

7. Murulana LC, Singh AK, Shukla SK, Kabanda MM, Ebenso EE. Experimental and quantum chemical studies of some bis (trifluoromethyl-sulfonyl) imide imidazolium-based ionic liquids as corrosion inhibitors for mild steel in hydrochloric acid solution. Industrial \& Engineering Chemistry Research. 2012;51(40):13282-99.

8. Zhao $\mathrm{H}$, Zhang $\mathrm{X}, \mathrm{Ji} \mathrm{L}, \mathrm{Hu} \mathrm{H}, \mathrm{Li}$ Q. Quantitative structure-activity relationship model for amino acids as corrosion inhibitors based on the support vector machine and molecular design. Corrosion Science. 2014;83:261-71.

9. Zhang D-Q, Cai Q-R, He X-M, Gao L-X, Zhou G-D. Inhibition effect of some amino acids on copper corrosion in $\mathrm{HCl}$ solution. Materials Chemistry and Physics. 2008;112(2):353-8.

10. Amin MA, Ibrahim MM. Corrosion and corrosion control of mild steel in concentrated $\mathrm{H} 2 \mathrm{SO} 4$ solutions by a newly synthesized glycine derivative. Corrosion Science. 2011;53(3):873-85.

11. Wazzan NA, Obot I, Kaya S. Theoretical modeling and molecular level insights into the corrosion inhibition activity of 2-amino-1, 3, 4thiadiazole and its 5-alkyl derivatives. Journal of Molecular Liquids. 2016;221:579-602.

12. Usman B, Jimoh I, Umar BA. THEORETICAL STUDY OF 2-(3, 4-DIHYDROXYPHENYL) CHROMAN$3,5,7-T R I O L$ ON CORROSION INHIBITION OF MILD STEEL IN ACIDIC MEDIUM. Applied Journal of Environmental Engineering Science.5(1):5-1 (2019) 66-74.

13. Atalay Y, Yakuphanoglu F, Sekerci M, AvCI D, Başoğlu A. Theoretical studies of molecular structure and vibrational spectra of 2-amino-5phenyl-1, 3, 4-thiadiazole. Spectrochimica Acta Part A: Molecular and Biomolecular Spectroscopy. 2006;64(1):68-72.

14. Ebenso EE, Arslan T, Kandemirli F, Caner N, Love I. Quantum chemical studies of some rhodanine azosulpha drugs as corrosion inhibitors for mild steel in acidic medium. International Journal of Quantum Chemistry. 2010;110(5):100318. 
15. Saha SK, Banerjee P. A theoretical approach to understand the inhibition mechanism of steel corrosion with two aminobenzonitrile inhibitors. RSC Advances. 2015;5(87):71120-30.

16. González-Olvera R, Espinoza-Vázquez A, Negrón-Silva GE, Palomar-Pardavé ME, RomeroRomo MA, Santillan R. Multicomponent click synthesis of new 1, 2, 3-triazole derivatives of pyrimidine nucleobases: Promising acidic corrosion inhibitors for steel. Molecules. 2013;18(12):1506479.

17. Viswanadhan VN, Ghose AK, Revankar GR, Robins RK. Atomic physicochemical parameters for three dimensional structure directed quantitative structure-activity relationships. 4. Additional parameters for hydrophobic and dispersive interactions and their application for an automated superposition of certain naturally occurring nucleoside antibiotics. Journal of chemical information and computer sciences. $1989 ; 29(3): 163-72$.

18. Arthur DE, Uzairu A, Mamza P, Abechi E, Shallangwa G. QSAR MODELLING OF SOME ANTICANCER PGI50 ACTIVITY ON HL-60 CELL LINES. Albanian Journal of Pharmaceutical Sciences. 2016;3(1):4-9.

19. Musa AY, Jalgham RT, Mohamad AB. Molecular dynamic and quantum chemical calculations for phthalazine derivatives as corrosion inhibitors of mild steel in $1 \mathrm{M} \mathrm{HCl}$. Corrosion Science. 2012;56:176-83.

20. Khaled K. Molecular simulation, quantum chemical calculations and electrochemical studies for inhibition of mild steel by triazoles. Electrochimica Acta. 2008;53(9):3484-92.

21. Nwankwo HU, Olasunkanmi LO, Ebenso EE. Experimental, quantum chemical and molecular dynamic simulations studies on the corrosion inhibition of mild steel by some carbazole derivatives. Scientific reports. 2017;7(1):2436.

22. Bereket G, Öğretir C, Özşahin Ç. Quantum chemical studies on the inhibition efficiencies of some piperazine derivatives for the corrosion of steel in acidic medium. Journal of Molecular Structure: THEOCHEM. 2003;663(1-3):39-46.

23. Bello A, Uzairu A, Shallangwa G. MOLECULAR MODELLING AND DYNAMIC SIMULATION OF CORROSION INHIBITORS ON STEEL IN ACIDIC MEDIUM. FUW Trends in Science \& Technology Journal. 2018;3 (2A):365-372

24. Ebenso EE, Khaled K, Shukla SK, Singh AK, Eddy N, Saracoglu M, et al. Quantum chemical investigations on quinoline derivatives as effective corrosion inhibitors for mild steel in acidic medium. 2012.

25. Abdallah M, Atwa S, Salem M, Fouda A. Synergistic effect of some halide ions on the inhibition of zinc corrosion in hydrochloric acid by tetrahydro carbazole derivatives compounds. Int J Electrochem Sci. 2013;8:10001-21.

26. Verma C, Olasunkanmi LO, Ebenso EE, Quraishi MA, Obot IB. Adsorption behavior of glucosamine-based, pyrimidine-fused heterocycles as green corrosion inhibitors for mild steel: experimental and theoretical studies. The Journal of Physical Chemistry C. 2016;120(21):11598-611.

27. Wazzan NA. DFT calculations of thiosemicarbazide, arylisothiocynates, and 1-aryl2, 5-dithiohydrazodicarbonamides as corrosion inhibitors of copper in an aqueous chloride solution. Journal of Industrial and Engineering Chemistry. $2015 ; 26: 291-308$.

28. Akalezi CO, Enenebaku CK, Oguzie EE. Application of aqueous extracts of coffee senna for control of mild steel corrosion in acidic environments. International Journal of Industrial Chemistry. 2012;3(1):13.

29. Shi W, Xia M, Lei W, Wang F. Molecular dynamics study of polyether polyamino methylene phosphonates as an inhibitor of anhydrite crystal. Desalination. 2013;322:137-43.

30. Zeng J, Zhang J, Gong X. Molecular dynamics simulation of interaction between benzotriazoles and cuprous oxide crystal. Computational and Theoretical Chemistry. 2011;963(1):110-4. 